\title{
Recent trends in the investigation of eating and its disorders
}

\author{
STEPHEN C. WOODS and ALFRED J. SIPOLS \\ University of Washington, Seattle, Washington
}

\begin{abstract}
In this paper, we summarize our views and evaluations of the papers presented at a symposium on recent trends in the study of eating and its disorders held at the meeting of the Psychonomic Society in New Orleans, 1990. We consider it an encouraging sign that the speakers focused to such a great extent upon experiential/associative/cognitive factors as being important determinants of human eating. In agreement with most of the speakers, we also feel that although great strides have been made in the attempt to understand the physiological influences over meals in well-controlled paradigms, there has been no significant application of this research to the study of human eating and its disorders. Perhaps more significant is that neither viable explanations for nor treatments of eating disorders based on physiological approaches have been forthcoming. The symposium provided excellent summaries of research on several important aspects of human eating and suggested areas in which research is needed.
\end{abstract}

The presentations and papers prepared for this symposium are both stimulating and refreshing. We found them to be stimulating because of the wealth of exciting new information that they provide, and because of the breadth of approaches being used to investigate food intake in general and human food intake in particular. We found them to be refreshing because of the emphasis on associative and cognitive factors that influence food intake. Most symposia on the controls of eating tend to focus on a particular neurotransmitter; or on the latest hot neuropeptide thought to be a satiety factor; or on some interesting subnucleus of the lower brainstem; or the like. It is relatively rare for the focus to be more on behavioral than on biological factors; and Betty Capaldi and the Psychonomic Society should be applauded for organizing this symposium with its excellent speakers. One might easily argue that our experience is biased by our particular interests, because the symposia that we usually attend are oriented more toward the physiological end of a continuum; but this explanation does not hold up. In an invited address at last year's meeting of the Eastern Psychological Association in Philadelphia, Terry Powley (1990) reported on an analysis that he had made of the most recent 25 years of peer-reviewed publications dealing with the topic of the controls of food intake. Although there was a clear trend for the absolute number of such publications to increase over years (reflecting a growing number of scientists in this field), the relative proportion of publications dealing with psychological or behavioral controls as op-

Preparation of this paper was aided by NIH Grant DK-17844. We thank Elizabeth Capaldi and the Psychonomic Society, and the other participants, for their help. Correspondence should be addressed to Stephen C. Woods, Department of Psychology, NI-25, University of Washington, Seattle, WA 98195. posed to more physiological ones was small and relatively constant (Powley, 1990).

The present symposium is especially timely because of what might be considered a failure of sorts on the part of many of us who study the physiology of eating to provide either viable explanations for or treatments of problems associated with human eating. This is particularly damning when one considers the eating disorders of anorexia nervosa and bulimia. For in spite of several decades of effort by the scientific community to identify an underlying physical cause of these disorders, a compelling case has yet to be made. Indeed, these disorders appear to be due to experiential and cultural, rather than organic, factors. Understanding these influences over food intake in general is therefore likely to be a fruitful approach to understanding eating disorders in particular; and the papers in this symposium provide a good starting point for much of what is known.

Those of us who investigate biological influences over feeding typically use a reductionistic approach. As an example, consider the ever-expanding literature on satiety hormones. These compounds, which often are small peptides, are secreted into the blood by the gastrointestinal system in response to eating and/or food in the gut; they contribute to the digestive process. In 1973, Gibbs, Smith, and their colleagues at the New York Hospital of Cornell University suggested that some of these compounds have the additional function of providing information to the nervous system about which or how much food has been eaten, and thereby contribute to the process of satiety; that is, when enough of these compounds have accumulated at critical sites within the body, a meal is terminated (Gibbs, Young, \& Smith, 1973).

This hypothesis has been widely embraced, and an extensive literature exists on the ability of one or another gut/digestive hormone to limit meal size in animals and 
humans (e.g., see Woods \& Gibbs, 1989). However, in most of these studies, meal size has been examined in isolation, in situations in which animals were slightly fooddeprived and trained to eat a meal of a particular size at a specified time. Deviations in meal size caused by the administration of some hormone can thus be easily detected, but the setting is quite unnatural in comparison with most instances of food consumption, especially among humans.

In one attempt to mimic a naturalistic eating situation more closely, we gave rats free access to food for $24 \mathrm{~h}$ a day (West, Fey, \& Woods, 1984). Whenever a rat initiated a meal, a remote pump was activated that delivered a small intraperitoneal infusion into the animal. This was all done automatically, with no intervention on the part of an experimenter. When the pump delivered a saline placebo solution, meal patterns were normal for all animals. However, when the pump delivered the putative satiety hormone, cholecystokinin (or CCK), at a dose that in other studies reduced the size of individual meals by half, a dramatic change occurred. Every meal that each rat consumed over a 6 -day interval was reduced, so that over the 6 days, meal size averaged around $50 \%$ of control values. At first glance, this would seem an ideal means of obtaining reduced food intake in individuals who need (or want) to lose weight. Unfortunately, during the same interval, the rats doubled the number of meals they initiated each day. So although the use of CCK could cause animals to eat smaller-than-normal meals, the animals compensated perfectly by eating more meals. The net result was a trivial and biologically insignificant change of body weight over the 6 days of treatment (West et al., 1984). It is our guess that if CCK or similar compounds were used therapeutically to reduce meal size in humans, a comparable phenomenon would occur, and the people would eat more meals or snack more often to make up the calories.

This discussion is not meant to imply that the research on satiety agents is not critical for understanding the controls of meal size or for suggesting potential therapeutic strategies; rather, we are simply pointing out that to date no therapeutic use has been made of this approach. To argue another side of the same coin, it is the case that several of these satiety agents, including CCK (Kissileff, Pi-Sunyer, Thornton, \& Smith, 1981), have been shown to reduce the size of individual meals among humans. Furthermore, when one considers the major clinical situation in which there is greatly altered meal size, bulimia, there is some correlative evidence that CCK physiology is altered. Geracioti and Liddle (1988) gave a group of bulimic women a standard meal of a fixed volume of a liquid diet. Weight and age-matched nonbulimic control women consumed an identical meal. The bulimic women reported feeling less sated (i.e., less full) after drinking the formula, and, importantly, they had a significantly smaller increase of blood levels of CCK after the meal than controls did, even though both groups had identical basal CCK levels. An inappropriately low endogenous satiety-signaling mechanism in bulimics could contribute to the syndrome of eating abnormally large meals. In the same study, following treatment of some of the bulimic women, the level of binging was reduced and the secretion of CCK into the blood after a test meal was normalized, further supporting a possible link between $\mathrm{CCK}$ and the syndrome.

Therefore, although it is fair to state that the physiological approach is shedding considerable light on the normal controls of eating, and although the potential for therapeutic applications is increasing, to date this potential has not been realized. Furthermore, there is growing evidence that associative processes play a major role in determining and influencing feeding, especially among humans. The next section provides an overview of the presentations made at the symposium.

\section{SYMPOSIUM OVERVIEW}

Neil Rowland, the initial speaker, points out that due to constraints of experimental design and subsequent interpretation, most studies of biological controls of food intake have necessarily been undertaken with reference to one or another single-factor model of homeostasis without consideration for learning effects or superimposable temporal factors such as nycthemeral rhythmicity (Rowland, 1991). Nevertheless, he feels that ample evidence has been generated to support the hypothesis that metabolic processes can play an influential role in the regulation of eating. Manipulating the local availability of oxidizable glucose, fructose, fatty acids, and ketones to specific central and peripheral sites can clearly, though sometimes only modestly, alter food intake. Furthermore, the failure of systemic manipulations to alter the magnitude of effects on feeding just as forcefully as local manipulations argues for an important influence of extrasystemic factors in the control of eating. These could include oral cues, gastrointestinal satiety signals, and learned or cognitive influences. Although brainstem nuclei have been proposed as possible locations for the integration of systemic and extrasystemic factors that is necessary to control eating behavior, much work remains to be done to elucidate the loci of such coordination and integration.

Rowland (1991) reminds us that the mediation of all neural processes, including information dealing with metabolic status, involves the coordination of multiple neurotransmitter systems, a number of which have been identified with respect to eating behavior. As an example, the orexigenic effects of norepinephrine and neuropeptide $\mathrm{Y}$ when administered into the paraventricular nucleus, and their reliance on adequate glucocorticoid levels from the adrenal glands, have been well documented (Leibowitz, 1988). Furthermore, these studies have begun to clarify specific aspects of feeding, such as carbohydrate preference, which might be critical for the understanding of eating disorders. The evidence for a physiological role of endogenous anorexigenic neurally active compounds such as insulin, CCK, corticotropin releasing factor, and sero- 
tonin is perhaps stronger than that for orexigenics, but little is known about the regulation of their synthesis and release in response to food intake.

Rowland (1991), in agreement with our own conclusion, notes that the studies of biological controls of eating have as yet failed to provide clear strategies in the treatment of human eating disorders, partly because of the lack of appropriate animal models of anorexia and bulimia. Obesity due to hyperphagia may be mildly attenuated in some cases by anorectic agents such as dexfenfluramine (Rowland \& Carlton, 1988), although there is no direct evidence to support a single neurotransmitter hypothesis in the etiology of human eating disorders.

According to Linda Bartoshuk, human studies of sensory controls of eating behavior underscore the necessary interplay of all sensory modalities involved in the perception and appreciation of foods (Bartoshuk, 1991). Temperature, pain, touch, taste, and olfaction combine to form unique sensory experiences, the preferences for which are highly susceptible to learning. While Davis's (1928) and Richter's (1942-1943) "wisdom of the body" may apply to a few nutrients such as sugar and small cations for which specific taste receptors and neural pathways exist, and for which an intrinsic avidity can be demonstrated in a novel deprivation paradigm, such innate deprivationdriven preference mechanisms are absent for the great majority of macro- and micronutrients. Recognition of protein, fat, starch, and vitamins in humans appears to be based solely on the learning of associations between specific foods and certain tastes and smells. This is either because the pure form of these nutrients is tasteless or because they are ingested in amounts too small to be tasted.

The hedonic properties of food, on the other hand, are innate with respect to the four basic tastes, with emotional affects associated with sweet, sour, salt, and bitter present at birth and universally understood. However, individual variability in sensory experience is ubiquitous. It arises from the heritability of sensory thresholds, from the effects of conditioning, and from physical deterioration (i.e., from age, damage, or disease) of structures involved in sensation and perception in a particular modality. For example, the hedonic aspects of olfaction can only be acquired through learning. In addition, genetic differences in (in)sensitivity to certain bitters and sweets, presumably due to simple Mendelian inheritance, have been well documented in studies of humans (e.g., Bartoshuk, 1979; Bartoshuk, Rifkin, Marks, \& Hooper, 1988; Gent \& Bartoshuk, 1983). Such differences may provide explanations for seemingly innate aversions to protein-rich foods like milk and cheese. Although species differences in sensitivity to bitter tastes, sodium, and aspartame are robust, it is unclear what light these differences shed on food preferences in people.

Bartoshuk (1991) points out that learning, then, clearly plays the major role in determining food intake based on sensory factors. Preferences for foods of a particular temperature, texture, taste, and smell are strongly conditioned to associated environmental cues such as time of day and location, and to social interactions and cognitive expectancies based on past experiences, with only salt and perhaps sugar having an additional inborn avidity mechanism upon which learning is superimposed.

The role of learning is further amplified in the paper by Anthony Sclafani (1991), which he was unable to present in person. (An oral presentation on learned food preferences was given instead by Robert Bolles.) Sclafani (1991) claims that it is not surprising that learned flavor aversions are readily formed in omnivores, including rats and humans, in which bitter substances innately appear to be the only actively avoided tastes amidst the tremendous variety and array of available foods. The ability to recognize the sensory properties of a toxic food that may have elicited unpleasant effects on previous exposure is critical to the survival of an animal. This robust learning phenomenon, based on close temporal associations of sensory stimuli with postingestive effects, can be formed after a single exposure, and it remains extremely resistant to extinction. Most real-world intake, however, has positive, not negative, postingestive consequences; yet even so, the ability to prefer foods on the basis of their nutritional benefits has not been as well characterized.

Rats exhibit innate preferences for the taste of salt, certain sweets including sugar, saccharin and starch, and the flavor of fat (Sclafani, 1987; see also Ackroff, Vigorito, \& Sclafani, 1989, cited in Sclafani, 1991). Sodium appetite appears unique in that it does not require prior conditioning in sodium-deprived states or previous oral exposure to salt for an appropriate ingestive response to hyponatremia. Some sort of postingestive nutritional feedback is required for other nutrients, and the conditioning of flavor preferences for nutritional solutions has only recently been demonstrated. For example, the pairing of neutral or mildly aversive nonnutritive cue flavors with intragastrically administered starch (Polycose) produces strong cue-flavor preferences in a number of paradigms with nondeprived animals (Sclafani \& Nissenbaum, 1988). On the other hand, intragastric fat (corn oil) infusions are effective in producing cue-flavor preferences only when the rats are food-deprived or initially trained with sweetened cue flavors (Lucas \& Sclafani, 1989, cited in Sclafani, 1991).

Given previous reports demonstrating the development of preferences for flavors associated with proteins (e.g., Baker, Booth, Duggan, \& Gibson, 1987), it is clear that postingestive feedback from a number of nutrients can shape subsequent eating patterns in the rat. In addition, there is evidence that these postingestive effects can be discriminated by the animal, in such a way that the rewarding nature of carbohydrates is much more pronounced than that of proteins. Whether a particular food's reinforcing quality is its caloric value, its satiating effects, or its ability to eliminate a nutritional deficit in the body (i.e., nutritive value), the neural and hormonal mechanisms mediating such conditioned flavor preferences via postingestive effects are poorly understood.

It is clear, therefore, that food choices and food intake in humans represent the integration of a number of physiological (e.g., metabolic and sensory) and psychological (e.g., cognitive and sociocultural) factors. Adam Drew- 
nowski (1991) believes that these may be different in normal-weight and obese individuals, and in those with eating disorders. The relative importance of these factors varies considerably among different populations, and probably within them as well.

The role of diet in the genesis and/or maintenance of a particular level of adiposity or of a clearly maladaptive eating behavior may be of critical importance. Drewnowski (1991) believes that this is especially true in light of preliminary evidence indicating an increased dietary fat (and reduced sugar) preference in overweight individuals with familial history of, early onset of, or repeated cycles of obesity, in comparison with normal-weight individuals (Drewnowski, Brunzell, Sande, Iverius, \& Greenwood, 1985). Anorectic patients, on the other hand, may display a decreased preference for dietary fat coupled with an avidity for sweets (Drewnowski, Halmi, Pierce, Gibbs, \& Smith, 1987).

The end result of these palatability differences (i.e., food intake reflective of such preferences) appears to be metabolically consistent with each disorder, since excess carbohydrates are dissipated as heat, whereas excess fat is more likely to be stored as adipose tissue. Therefore, although learning and conditioning clearly play a role in determining such taste preferences, Drewnowski (1991) agrees with Rowland (1991) that the evidence for altered neurotransmission as the primary cause of enhanced carbohydrate intake and/or bulimic or anorectic behavior (e.g., as has been variously suggested for serotonin, neuropeptide $\mathrm{Y}$, endogenous opiates, and CCK) is tenuous at best.

Drewnowski (1991) points out that a number of cognitive and experiential factors (e.g., dietary habits, sociocultural attitudes, and perceptions of body appearance) may disrupt the close correspondence between food responsiveness, acceptability, and ingestion, so that initial palatability may not necessarily lead to increased intake (Mattes, 1985). Nevertheless, evidence from a number of epidemiological studies strongly points to a positive association between obesity and dietary fat intake and a negative correlation with sugar intake. Anorectics have an aversion to dietary fats while carbohydrate intake remains normal; bulimics typically binge on high-fat, moderatecarbohydrate, low-protein foods (Drewnowski, Pierce, \& Halmi, 1988). Elucidating the precise origin of such taste preferences, which appear to be based on a learning paradigm tempered by metabolic feedback, remains a difficult task, hampered, as Rowland (1991) mentions, by the lack of appropriate animal models of anorexia and bulimia.

Leann Birch (1991) pointed out that the transformation from consummatory behavior in infancy, when all nutritional requirements are satisfied by milk, to omnivorous intake in childhood and beyond, when dietary variety is necessary for adequate nutrition, is a poorly understood phenomenon. Yet despite the tremendous variety among and between cultures, energy balance in the adult is usually maintained, with eating occurring as a result of complex physiological and psychological interactions, usually in the absence of any obvious nutritional deficits. Overnutrition (i.e., as in obesity) is an increasingly preva- lent problem along with anorexia and bulimia; therefore, to elucidate how food preferences, which may determine diet and food selection in later years, develop in early childhood will provide an important contribution toward our understanding of the learning and experiential antecedents of eating disorders.

Studies of single-meal intake patterns have yielded clear evidence that infants and young children, in contrast with adults, are relatively responsive to the energy density of foods (Birch \& Dysher, 1985). Furthermore, after repeated experience with foods differing only in energy density, children develop a preference for energy-dense foodstuffs, demonstrating the powerful conditioning effects of postingestive nutritional consequences (Birch, McPhee, Steinberg, \& Sullivan, 1990). Conversely, the satiating effects of calorically dense foods have also been demonstrated to cause aversions to flavors associated with them. Perhaps in part because of these physiological cues, total daily energy intake by young children (in the absence of adult intervention) appears to be relatively tightly regulated through compensation across successive meals, notwithstanding highly variable individual meal intake (Birch, Johnson, Andresen, Peters, \& Schulte, 1991).

Owing to the social (e.g., parental) context in which eating patterns are formed and to the redundant nature of eating, which provides endless opportunities for associative conditioning, psychological factors are paramount in the development of food acceptance patterns. There is evidence that a child's commonly observed preference for "inappropriate" foods at the expense of "healthy" foods is due to eating practices instituted by parents. Children have been shown to learn to dislike foods that they are rewarded for eating (Birch, Marlin, \& Rotter, 1984), and foods high in fat, sugar, or salt are often used as rewards themselves. In addition, children seem to develop preferences for familiar foods as they develop a dislike for unfamiliar versions, regardless of the flavor of each.

This familiarity can be extended to external signals. Meal initiation in children can be shown to be stimulated by contextual cues in the absence of nutritional cues (Birch, 1991), a learning phenomenon that contrasts with the depletion-driven eating of infancy. Other external cues that can modify a child's responsiveness to internal signals, and hence his or her feelings of hunger and satiety, include time of day, amount of food remaining, rewards for eating, etc.

Therefore, the internal feedback that controls food intake so well in infancy is subject to many associative conditioning (e.g., social) influences during childhood. Eventually, these influences minimize the role of internal signals and effect the formation of food preferences that can be very unique in a population and different between populations.

In light of the presentations, we offer the model depicted in Figure 1 as a contemporary guide to the influences over human food intake. The underlying concept is that different types and levels of controls influence individual meals rather than intake over larger intervals, and that many fac- 


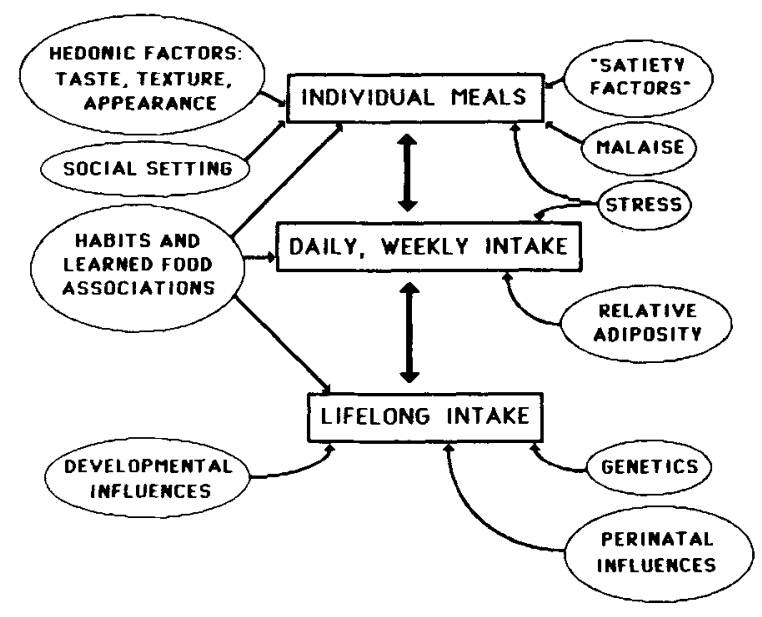

Figure 1. Influences over food intake.

tors superimpose life-long controls. We think that this model will enable new information on the possible controls of eating disorders to be better understood, especially models of eating disorders based on addictive personalities and/or cultural and familial attitudes toward body shape.

\section{AN ASSOCIATIVE CHALLENGE}

In reading over the papers presented in this symposium, and in considering their emphasis on the role of learning in determining and influencing human food intake patterns, we were reminded of a rather unique eating experience that we had a few years ago. We relate it here because we think that it represents a challenge of sorts for many of the symposium speakers, and we suggest it as a potential research topic (for others!) that evidently remains untapped. It has to do with the durian, a large (pineapple-sized) pithy fruit indigenous to Southeast Asia, which falls from trees when it ripens. The durian is believed by some natives to be an aphrodisiac, and so it is widely sought after (at least by a subset of the local inhabitants). The durian was brought to our personal attention by Scott Parker of American University, who seeks it out because he finds it palatable. The durian is, in fact, well known for its chemosensory qualities. What is particularly interesting for present consideration is the distinctly opposite nature of hedonic experiences generated by the durian in different individuals, coupled with the degree or severity of their reactions. Upon Parker's recommendation, we and several colleagues once ordered and sampled a durian milkshake at a Vietnamese restaurant in Toronto several years ago. We had been apprised (somewhat) of its powerful chemosensory effect, but we were clearly not prepared for the impact. We should point out that all seven of us who were present at that meal were naive with respect to the durian, but that we are often quite adventuresome in our selection of food. We had all agreed a priori to sample at least one mouthful of the milkshake, which we did somewhat simultaneously. Never have we witnessed such a universal response of disgust. To a person, we gagged and wished we had never put the stuff in our mouths. Some of us actually did swallow some, but we all felt disgusted. To this day, each of us can vividly recall the taste/smell of the durian, and even the suggestion of having to consume one generates this response of disgust. Surely this is a strong reaction.

The durian has an aroma and flavor described by various food writers as "foul," "pungent, like overripe cheese," "resembling an open sewer," and so forth, not to mention other, less flattering, comments. Others, aficionados, describe it as "subtly exotic," "the most delicious of tropical fruits," etc. Why such a vast difference? Certainly many foodstuffs are inherently unpalatable yet come to be tolerated or even preferred with repeated experience (e.g., see Rozin \& Fallon, 1987). But it is not clear that the initial response (we hesitate to use the term "unconditioned") in such cases is one of such complete disgust (nor do we know if very young children would find the durian disgusting). The durian is sufficiently pungent that in many Southeast Asian countries it is barred from any form of public transportation (e.g., buses, subways, airplanes), and in fact it cannot be legally brought into some countries. We know of no other foodstuff for which there exists specific legislation based solely on its chemosensory properties. It would seem an ideal topic for investigation by scientists interested in developmental and learned preferences for foods, in the response of the gustatory system to foods and how this translates into palatability, and in the unconditioned responses to particularly unique foodstuffs.

\section{CONCLUSION}

In summary, it is fair to state that just as considerable knowledge is being generated on the role of specific nutrients, hormones, neurotransmitters, and the like, on food intake, there is also a growing trend toward consideration of the role of associative factors. Figure 1 depicts our view of this trend and of how the various controls over feeding might be considered to interact.

\section{REFERENCES}

BAker, B. J., Booth, D. A., Duggan, J. P., Gibson, E. L. (1987). Protein appetite demonstrated: Learned specificity of protein-cue preference to protein need in adult rats. Nutrition Research, 7, 481-487.

BARTOSHUK, L. M. (1979). Bitter taste of saccharin: Related to the genetic ability to taste the bitter substance 6-N-propylthiouracil (PROP). Science, 205, 934-935.

BARTOSHUK, L. M. (1991). Sensory factors in eating behavior. Bulletin of the Psychonomic Society, 23, 250-255.

Bartoshuk, L. M., RifKin, B., MARKs, L. E., \& Hooper, J. E. (1988). Bitterness of $\mathrm{KCl}$ and benzoate: Related to PTC/PROP. Chemical Senses, 13, 517-528.

BirCH, L. L. (1991). Obesity and eating disorders: A developmental perspective. Bulletin of the Psychonomic Society, 23, 265-272.

BirCH, L. L., \& Dysher, M. (1985). Conditioned and unconditioned caloric compensation: Evidence for self-regulation of food intake by young children. Learning \& Motivation, 16, 341-355.

Birch, L. L., Johnson, S. L., Andresen, G., Peters, J. C., 
Schulte, M. C. (1991). The variability of young children's energy intake. New England Journal of Medicine, 324, 232-235.

BIRCH, L. L., MARLIN, D., \& RotTER, J. (1984). Eating as the "mean" activity in a contingency: Effects on young children's food preference. Child Development, 55, 532-539.

Birch, L. L., McPhee, L., Steinberg, L., \& Sullivan, S. (1990). Conditioned flavor preferences in young children. Physiology \& Behavior, 47, 501-505.

DAvis, C. M. (1928). Self selection of diet by newly weaned infants: An experimental study. American Journal of Diseases of Children, 36, 651-679.

DrewNowskI, A. (1991). Obesity and eating disorders: Cognitive aspects of food preference and food aversion. Bulletin of the Psychonomic Society, 23, 261-264.

Drewnowski, A., Brunzell, J. D., Sande, K., Iverius, P. H., \& GREENWOOD, M. R. C. (1985). Sweet tooth reconsidered: Taste preferences in human obesity. Physiology \& Behavior, 35, 617-622.

Drewnowski, A., Halmi, K. A., Pierce, B., Gibbs, J., \& Smith, G. P. (1987). Taste and eating disorders. American Joumal of Clinical Nutrition, 46, 442-450.

Drewnowski, A., Pierce, B., \& Halmi, K. A. (1988). Fat aversion in eating disorders. Appetite, 10, 119-131.

GeNT, J. F., \& BARToshuk, L. M. (1983). Sweetness of sucrose, neohesperidin dihydrochalcone and saccharin is related to genetic ability to taste the bitter substance 6-N-propylthiouracil. Chemical Senses, 7, 265-272.

Geracioti, T. D., JR., \& LIDDLE, R. A. (1988). Impaired cholecystokinin secretion in bulimia nervosa. New England Journal of Medicine, 319, 683-688.

Gibes, J., Young, R. C., \& Smith, G. P. (1973). Cholecystokinin decreases food intake in rats. Journal of Comparative \& Physiological Psychology, 84, 488-495.

KissilefF, H. R., Pi-Sunyer, F. X., Thornton, J., \& Smith, G. P.
(1981). C-terminal octapeptide of cholecystokinin decreases food intake in man. American Journal of Clinical Nutrition, 34, 154-160.

LEIBowITZ, S. F. (1988). Hypothalamic paraventricular nucleus: Interaction between alpha 2-noradrenergic system and circulating hormones and nutrients in relation to energy balance. Neuroscience \& Biobehavioral Reviews, 12, 101-109.

MATtEs, R. D. (1985). Gustation as a determination of ingestion: Methodological issues. American Journal of Clinical Nutrition, 41, 672-683.

Powley, T. L. (1990, March). The psychobiology of feeding and satiety circa 1990: Taking stock of directions, past, present and future. Paper presented at the meeting of the Eastern Psychological Association, Philadelphia.

RiChTER, C. P. (1942-1943). Total self regulatory functions in animals and human beings. Harvey Lecture Series, 38, 63-103.

Rowland, N. E. (1991). Biological factors in eating and its disorders. Bulletin of the Psychonomic Society, 23, 244-249.

Rowland, N. E., \& Carlton, J. (1988). Dexfenfluramine: Effects on food intake in various animal models. Clinical Neuropharmacology, 11(Suppl. 1), S33-S50.

Rozin, P., \& Fallon, A. E. (1987). A perspective on disgust. Psychological Review, 94, 23-41.

SClaFANI, A. (1987). Carbohydrate taste, appetite, and obesity: An overview. Neuroscience \& Biobehavioral Reviews, 11, 131-153.

SClafani, A. (1991). Conditioned food preferences. Bulletin of the Psychonomic Society, 23, 256-260.

SClAFANI, A., \& Nissenbaum, J. W. (1988). Robust conditioned flavor preference produced by intragastric starch infusions in rats. American Journal of Physiology, 255, R672-R675.

WeST, D. B., FEY, D., \& Woods, S. C. (1984). Cholecystokinin persistently suppresses meal size but not food intake in free-feeding rats. American Journal of Physiology, 246, R776-R787.

Woods, S. C., \& Gibss, J. (1989). The regulation of food intake by peptides. Annals of the New York Academy of Sciences, 575, 236-243. 